\title{
A molecularly imprinted polymer coated-nanocomposite of magnetic nanoparticles for organic compounds recognition
}

\author{
Maria Guć1, Mateusz Pawlaczyk ${ }^{1}$, Grzegorz Schroder ${ }^{1}$ \\ ${ }^{1}$ Adam Mickiewicz University in Poznan, Faculty of Chemistry, \\ Umultowska 89b, 61-614 Poznań, Poland \\ maria.guc@amu.edu.pl; mateusz.pawlaczyk@amu.edu.pl; schroede@amu.edu.pl
}

\begin{abstract}
Following article presents an efficient method for the core-shell systems preparation, composed of magnetic nanoparticles modified with molecularly imprinted polymers (mag-MIP). Obtained mag-MIP were utilized for pre-concentration and trace analysis of organic compounds in real samples. Superparamagnetic iron oxide nanoparticles (SPION) modified with TEOS (tetraethoxysilane) and MPS (3-(Trimethoxysilyl)propyl methacrylate) were used as the magnetic core, EGDMA (ethylene glycol dimethacrylate) was used as a cross-linking agent and AIBN (2,2'-azobisisobutyronitrile) as a thermal polymerisation initiator. Quercetin, estrone and $\beta$-estradiol were used as templates for which the appropriate monomers were chosen. Mag-MIP were successfully applied for the determination of chemical compounds in environmental samples. Quercetin, estrone and $\beta$-estradiol were adsorbed from their solutions onto the surface of functionalised SPION. Subsequently, mag-MIP were attracted by magnets immersed in the solutions and analysed via electrospray ionization mass spectrometry (ESI-MS) and flowing atmospheric pressure afterglow mass spectrometry (FAPA-MS) combined with thermally initiated desorption. The desorption occurs concomitantly with the plasma stream ioniaztion of the molecules, which are then transported to the analyser. FAPA-MS combined with mag-MIP is a novel analytical method suitable for trace detection from highly heterogeneous solutions. The combination of analyte pre-concentration with mag-MIP followed by FAPA-MS analysis significantly reduced limit of detection (LOD) for several trace analyses.
\end{abstract}

Keywords: Superparamagnetic nanoparticles $\left(\mathrm{Fe}_{3} \mathrm{O}_{4}\right)$, nanomaterials, molecularly imprinted polymers (MIP), magnetic molecularly imprinted polymers (mag-MIP), sample pretreatment, trace analysis, ESI-MS, FAPA-MS.

\section{Introduction}

Shortened sample preparation protocol for quantitative and qualitative determination of analytes is one of the key tasks of contemporary analytical chemistry. One of the approaches involves the use of selective solid-phase extraction (SSPE) systems in combination with mass spectrometry. SSPE may utilize molecular imprinted polymers (MIP), which allows for sample pre-concentration [1-2]. MIP and their magnetic derivatives, containing magnetic nanoparticle as a core, (mag-MIP) production is based on the formation of complexes between the analyte/template/guest molecule and the functional monomer/host molecule. This technique involves polymerization of functional monomers and a cross-linker around a template. The analyte-monomer complex is then subjected to polymerization initiated by temperature or irradiation (thermal or photoinitiation, respecitvely) during which a three-dimensional polymer structure is formed. Subsequently, the template is removed from the polymeric structure, what generates empty cavities capable of binding the template molecules as they are complementary in size, shape and functional group positions. The use of organic porogens makes polymers hydrophobic. The polymers obtained were chemically stable, water insoluble, what permits for their multiple use in chemical analysis and the synthesis process is relatively simple, reproducible and cheap. MIP as a nanomaterial, have a better performance for selective removal of trace analytes from complex samples because of its small particle size and high specific surface area what leads to the high adsorption capacity and fast adsorption rate [3-5]. Thus, molecularly imprinted polymers have various potential applications e.g.: amino acid and protein recognition, pesticides, drugs, endocrine disrupting chemicals detection etc. In more recent applications, mag-MIP were used for the recognition of organic compounds in environmental samples and performed better in comparison to MIP [6-7]. Obtained mag-MIP retain their magnetic properties, and thus can still be manipulated easily by external magnetic fields. Additionally, the core provides defined particle size and shape which results in a higher surface area in comparison to MIP [8-9]. Moreover, the recognition sites are located on the surface of the material, facilitating the analyte binding. Due to their magnetic and surface properties, mag-MIP are more efficient than MIP obtained 


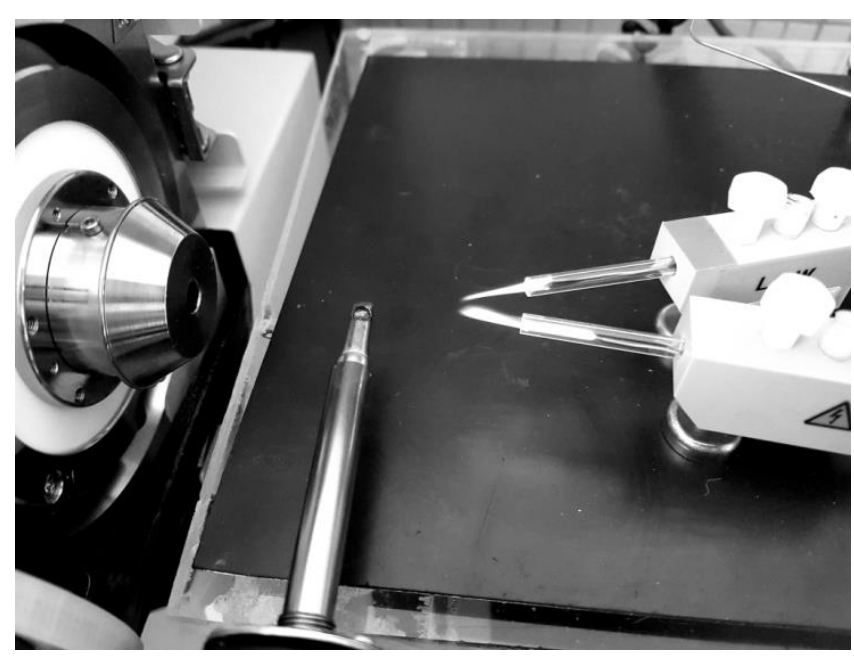

Fig. 1: The system used to carry out the experiment: a) mass spectrometer inlet, b) heating system,

c) FAPA-ion source.

by traditional synthesis methods. These advantages of magMIP render them highly attractive for a wide variety of applications in separation techniques [10-11]. Ambient ionization mass spectrometry comprises a group of various techniques, which allow for MS analysis at atmospheric pressure. They provide rapid, direct, and high-throughput analyses with no or only minimal sample pre-treatment. Moreover, substances can be analysed directly from surfaces or other matrices. They involve the generation of a direct current or radiofrequency electrical discharge between a pair of electrodes in contact with a flowing inert gas, creating a stream of ionized molecules, radicals, excited state neutral atoms, and electrons. The plasma species are directed towards the sample, resulting in desorption and ionization of the analyte. Ambient plasma MS techniques have many advantages due to their simple instrumentation, rugged construction, absence of solvents, and generation of singly charged analyte species that are easier identifiable than multiple charged ions and various adducts produced by the

spray based techniques [12].

This paper presents new analytical procedures for determination of organic compounds: quercetin, estrone and $\beta$-estradiol in real samples with the use of mag-MIP for pre-concentration and flowing atmospheric-pressure afterglow mass spectrometry (FAPA-MS) with thermal desorption of analytes. The proposed method was compared with ESI-MS analysis of quercetin or hormones in water solutions. The combination of analyte pre-concentration technique with mag-MIP and FAPA-MS analysis created new method with decreased LOD for several trace analytes.

\section{Experimental}

\subsection{Materials and chemicals}

All reagents used were commercial products. $\mathrm{FeCl}_{2} \cdot 4 \mathrm{H}_{2} \mathrm{O}, \mathrm{FeCl}_{3} \cdot 6 \mathrm{H}_{2} \mathrm{O}$, tetraethoxysilane (TEOS), hydrochloric acid $(\mathrm{HCl})$, citric acid, sodium hydroxide $(\mathrm{NaOH})$, ammonia solution $\left(\mathrm{NH}_{4} \mathrm{OH}\right)$, acrylamide (AA), methacrylic acid (MA), ethylene glycol dimethacrylate (EGDMA), 2,2'-azobisisobutyronitrile solution $0.2 \mathrm{M}$ in toluene (AIBN), Aluminum Oxide Activated, basic, Brockman I, 3-metacriloxipropiltrimetoxissilano (MPS), quercetin (Q), estrone (E1), $\beta$-estradiol (E2) and all solvents: toluene, chloroform, N,N-Dimethylformamide (DMF), ethanol, acetic acid of the purity grade p.a., were obtained from Sigma-Aldrich (St. Louis, MO, USA).

\subsection{Instruments}

The material was tested by Fourier Transform Infrared (FTIR) spectroscopy and thermogravimetric analysis (TGA). The infrared spectra were taken on an IFS 66 v/s FTIR spectrophotometer from Bruker, equipped with an MCT detector (125 scans, resolution $2 \mathrm{~cm}^{-1}$ ). The spectra were recorded in the $400-4000 \mathrm{~cm}^{-1}$ range for $\mathrm{KBr}$ pellets. Functionalization of the materials was confirmed via TGA. The measurements were performed on Setsys 1200 thermogravimetric analyzer (Setaram) with a heating rate of $10^{\circ} \mathrm{C} \mathrm{min}^{-1}$ from room temperature to $1000^{\circ} \mathrm{C}$ in helium atmosphere. Scanning electron microscopy (SEM) images were acquired with QUANTA 250 FEG, FEI.

ESI-MS and ESI-MS ${ }^{n}$ spectra were recorded using amaZon SL ion trap (Bruker, Bremen, Germany) equipped with electrospray ion source in infusion mode. The sample solution was introduced into the ionization source at a flow rate of $5 \mu \mathrm{L} \mathrm{min}{ }^{-1}$ using a syringe pump. The apparatus was operated using the so-called "enhanced resolution mode" (mass range: $50-2,200 \mathrm{~m} / z$, scanning rate: $8,100 \mathrm{~m} / \mathrm{z}$ per second). The capillary voltage was set at $-4.5 \mathrm{kV}$ and the endplate offset at $-500 \mathrm{~V}$. The source temperature was $80^{\circ} \mathrm{C}$ and the desolvation temperature was $250^{\circ} \mathrm{C}$. Helium was used as the cone 
gas and desolvating gas (nitrogen) at flow rates of $50 \mathrm{Lh}^{-1}$ and $800 \mathrm{Lh}^{-1}$, respectively. The mass spectrometer was operated in the ESI positive and negative ionization mode. In $\mathrm{MS}^{n}$ experiments, the width of the selection window was set at $2 \mathrm{Da}$ and and the amplification of the excitation was set according to the experiment (from 0.2 to $1.5 \mathrm{~V}$ ).

Mass spectrometers were equipped optionally with FAPA ambient plasma source (ERTEC, Wroclaw, Poland). Experimental details for the FAPA method were presented in our previous publication [12].

\subsection{Synthesis of magnetic molecularly imprinted polymers}

Mag-MIP were synthesized by the modified method described in literature [1]. The magnetite nanoparticles $\left(\mathrm{Fe}_{3} \mathrm{O}_{4}\right)$ were synthesized in a two-step procedure. Firstly, magnetite $\left(\mathrm{Fe}_{3} \mathrm{O}_{4}\right)$ was synthesized by the precipitation method by using $\mathrm{FeCl}_{2} \cdot 4 \mathrm{H}_{2} \mathrm{O}$ and $\mathrm{FeCl}_{3} \cdot 6 \mathrm{H}_{2} \mathrm{O}$ as ferric and ferrous ions precursors [9]. $\mathrm{FeCl}_{2} \cdot 4 \mathrm{H}_{2} \mathrm{O}(2 \mathrm{~g})$ and $\mathrm{FeCl}_{3} \cdot 6 \mathrm{H}_{2} \mathrm{O}(5.2 \mathrm{~g})$ were dissolved into $25 \mathrm{ml}$ deoxygenated water, and then $0.85 \mathrm{ml}$ of concentrated $\mathrm{HCl}$ were added. The resulting solution was added dropwise into $250 \mathrm{ml}$ of $1.5 \mathrm{M} \mathrm{NaOH}$ solution upon vigorous stirring in $\mathrm{N}_{2}$ atmosphere at $80^{\circ} \mathrm{C}$. The synthesized magnetic nanoparticles (MNPs) were separated from the solution with a magnet and washed with 200 ml of deionized water three times. Then, the surface-functionalized magnetite was produced via hydrolysis and condensation of the TEOS organosilane agents. Firstly, $10 \mathrm{ml}$ of an aqueous solution of citric acid $(0.5 \mathrm{~g} / \mathrm{ml})$ was added to the rigorously stirred suspension of washed nanoparticles. The $\mathrm{pH}$ value was set to 5.2 with concentrated ammonia solution and heated to $80^{\circ} \mathrm{C}$. After 90 mins, the $\mathrm{pH}$ value of the solution was elevated to 10, using $\mathrm{NaOH}$ solution. Lastly, the suspension was centrifuged for $5 \mathrm{mins}$ at $4000 \mathrm{rpm}$ to remove any agglomerated nanoparticles. Next, silica coat was fabricated on the surface of $\mathrm{Fe}_{3} \mathrm{O}_{4}$ MNPs through a sol-gel method. $100 \mathrm{ml}$ of ethanol containing $4 \mathrm{ml}$ of TEOS were added in the above prepared stable aqueous suspension of $\mathrm{Fe}_{3} \mathrm{O}_{4} \mathrm{MNPs}$, followed by stirring at ambient temperature for $6 \mathrm{~h}$. After rinsing with ethanol and water several times, the obtained $\mathrm{Fe}_{3} \mathrm{O}_{4} @ \mathrm{SiO}_{2} \mathrm{MNPs}$ were dried at $60^{\circ} \mathrm{C}$. In the next step, $\mathrm{Fe}_{3} \mathrm{O}_{4} @ \mathrm{SiO}_{2}$ was modified with MPS. For this purpose $250 \mathrm{mg}$ of obtained material were dispersed in $50 \mathrm{~mL}$ of anhydrous toluene containing $5 \mathrm{~mL}$ of MPS and the mixture was allowed to react at $60^{\circ} \mathrm{C}$ for $24 \mathrm{~h}$ under dry nitrogen. The mixture was filtered through a membrane, washed with toluene and dried in vacuum. Finally, the silanized and modified material $\mathrm{Fe}_{3} \mathrm{O}_{4} @ \mathrm{SiO}_{2}-\mathrm{MPS}$ was obtained. The next step involved

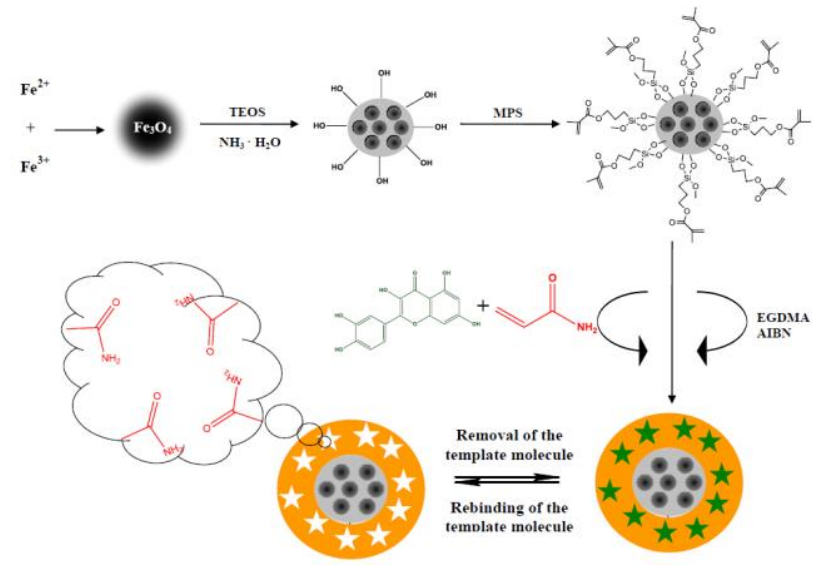

Fig. 2: General steps for the preparation of magnetic molecularly imprinted polymer selective for quercetin (Q-mag-MIP). the preparation of a selective mag-MIP towards chosen analyte. For instance, mag-MIP selective for quercetin was prepared by polymerization of $0.2 \mathrm{mmol}$ template-Q and $0.8 \mathrm{mmol}$ functional monomer-AA in $30 \mathrm{~mL}$ of a porogenic solvent - ethanol. The mixture was placed in ultrasound bath at $25^{\circ} \mathrm{C}$ for $1 \mathrm{~h}$. Then, $200 \mathrm{mg}$ of $\mathrm{Fe}_{3} \mathrm{O}_{4} @ \mathrm{SiO}_{2}$-MPS were added into the mixture, which was sonicated for another $1 \mathrm{~h}$. Subsequently, $4.0 \mathrm{mmol}$ of the cross-linking agentEGDMA and $1 \mathrm{ml}$ of the initiator-AIBN were added into the system and the mixture was sonicated at $80^{\circ} \mathrm{C}$ for $24 \mathrm{~h}$. Mag-MIP selective for hormones (estrone, $\beta$-estradiol) were prepared in the same manner, by polymerization of $1 \mathrm{mmol}$ template-E1 or E2 and $4 \mathrm{mmol}$ functional monomer-MA in $30 \mathrm{~mL}$ of a same porogenic solventethanol. The mixture was placed in ultrasound bath at $25^{\circ} \mathrm{C}$ for $1 \mathrm{~h}$. Then $200 \mathrm{mg}$ of $\mathrm{Fe}_{3} \mathrm{O}_{4} @ \mathrm{SiO}_{2}$-MPS were added into the system and the mixture was sonicated for another 1h. Next, $20 \mathrm{mmol}$ of the crosslinking agent-EGDMA and $1 \mathrm{ml}$ of the initiator-AIBN were added into the system and the mixture was sonicated at $80^{\circ} \mathrm{C}$ for $24 \mathrm{~h}$. All obtained materials: Q-mag-MIP, E1-mag-MIP, E2-mag-MIP were dried under reduced pressure, and grounded using mortar and pestle. Afterwards, the templates were removed by Soxhlet extraction using a mixture of ethanol: acetic acid (9:1, v:v) as eluent for 7 days. The eluent was exchanged every 2 days. Finally, mag-MIP selective for: Q, E1, E2 were dried and grounded.

The levels of template elution were controlled with two techniques: ESI-MS analysis of solution after extraction and the FAPA-MS analysis of the dry mag-MIP. It was determined that after the extraction, materials containing about $1 \%$ and $5 \%$ 
of a template in the mag-MIP were obtained. Further extraction or solvent substitution did not change the template elution level.

As a control experiment, magnetic non-imprinted polymers (mag-NIP) were also prepared and treated in the identical manner, excluding utilization of template structures during polymerisation process.

\subsection{Trace analysis of organic compounds}

To establish the concentration range suitable for qualitative and quantitative ESI-MS determination of organic compounds in solutions, serial dilutions were prepared. In the range of negative or positive ions, a series of 10 standard water solutions of these compounds were prepared in which the concentration of quercetin, estrone, $\beta$-estradiol varied from $10^{-4}$ to $10^{-9} \mathrm{M}$. The LOD and linearity range were determined for each compound. The same procedure was used for the FAPA-MS analysis. The combination of analyte pre-concentration with mag-MIP followed by FAPA-MS analysis significantly reduced LOD for several trace analyses. For this purpose, $10 \mathrm{mg}$ of mag-MIP were placed in $10 \mathrm{ml}$ of the analyzed solution, where the process of the analyte adsorption to selective polymeric cavities occurs. Mag-MIP with the adsorbed analyte was isolated with neodymium magnet and placed directly in the plasma stream of FAPA-MS.

\section{Results and discussion}

3.1. Characterization of Q-mag-MIP, E1-mag-MIP, E2-mag-MIP

\subsubsection{FTIR spectra}

We performed FTIR analysis of Q-mag-MIP, E1-mag-MIP, E2-mag-MIP and mag-NIPs. A strong and broad absorbance peak assigned to stretching vibration of hydroxyl groups were found at $3300 \mathrm{~cm}^{-1}$. Hydroxyl groups in the

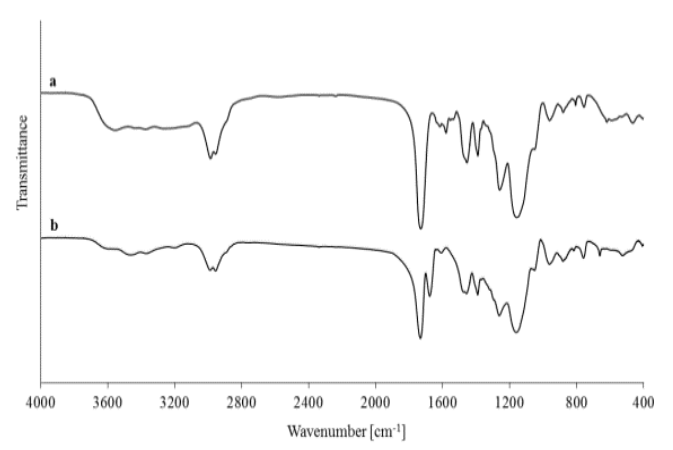

Fig. 3: The FTIR spectra of (a) mag-NIP, (b) Q-mag-MIP. quercetin and hormones formed intra- and/or inter-molecular hydrogen bonds. The peak corresponding to the vibrations of the carbonyl group was found at $1656 \mathrm{~cm}^{-1}$. In addition, peaks assigned to the quercetin molecule were found at $1615 \mathrm{~cm}^{-1}, 1512 \mathrm{~cm}^{-1}$ and $1432 \mathrm{~cm}^{-1}$. For AA monomer the main absorption bands at around $3356 \mathrm{~cm}^{-1}, 1673 \mathrm{~cm}^{-1}$, and $1613 \mathrm{~cm}^{-1}$ were associated with the following vibrations: N-H stretching, (being characteristic absorption band for primary amine in acrylamide), $\mathrm{C}=\mathrm{O}$ stretching and $\mathrm{C}=\mathrm{C}$ stretching, respectively. The FTIR spectra of mag-NIPs were utilized as a useful comparative tool. For Q-mag-MIP and Q-mag-NIP strong peaks at 1738

$\mathrm{cm}^{-1}$ and $1733 \mathrm{~cm}^{-1}$ were attributed to $\mathrm{C}=\mathrm{O}$ stretching vibration, while peak at 1678 $\mathrm{cm}^{-1}$ was observed only for Q-mag-MIP and was attributed to the $\mathrm{C}=\mathrm{O}$ stretching vibration from quercetin. The broad peak at $2830-3696 \mathrm{~cm}^{-1}$ was assigned to the asymmetric stretching of $\mathrm{N}-\mathrm{H}$ and hydrogen-bonded interaction between quercetin molecules and amid groups. For Q-mag-MIP, E1-mag-MIP, E2-mag-MIP and mag-NIP strong peaks at $1078 \mathrm{~cm}^{-1}, 1099$ $\mathrm{cm}^{-1}, 1095 \mathrm{~cm}^{-1}$ and $1097 \mathrm{~cm}^{-1}$ were observed, which were attributed to the stretch of Si-O-Si, indicating the formation of silica film. The typical peak of $1741 \mathrm{~cm}^{-1}$ and $1737 \mathrm{~cm}^{-1}$ represent the stretching vibration of carbonyl groups of estrone and $\beta$-estradiol, respectively. In addition, the most intense

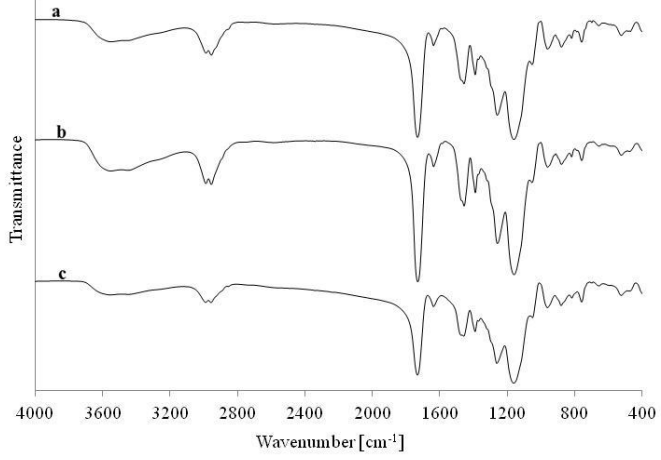

Fig. 4: The FTIR spectra of (a) E2-mag-MIP, (b) E1-mag-MIP, c) mag-NIP. hydrogen bonds $3200-3600 \mathrm{~cm}^{-1}$ are formed between the monomers and the estrone molecules in E1-mag-MIP, slightly less intense between $\beta$-estradiol and the monomer molecules, and the least intense in the polymer structure itself. This confirms the introduction of individual hormones into polymeric structures. 


\subsubsection{Thermal analysis}

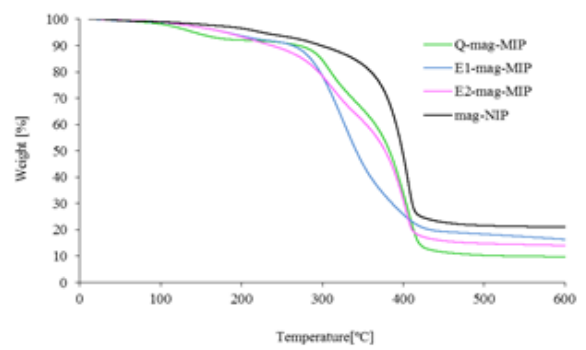

mag-MIP, E1-mag-MIP, E2-mag-MIP and mag-NIP.
Q-mag-MIP, E1-mag-MIP, E2-mag-MIP and the corresponding mag-NIP materials exhibit similar thermal properties. The polymers are stable up to $250^{\circ} \mathrm{C}$, where only a small mass loss was observed related to the water loss from the polymer structure. The greatest mass loss was observed in a $300-430^{\circ} \mathrm{C}$ range. The loss was more pronounced for Q-mag-MIP than for mag-NIP, which signifies the release of quercetin from the materials (melting point of quercetin is $316^{\circ} \mathrm{C}$ ). The most intensive desorption of quercetin was observed in a $330-350^{\circ} \mathrm{C}$ range. These temperatures were applied for thermal desorption of the analyte from Q-mag-MIP in FAPA-MS analysis. Similar results were obtained for nanomaterials selective for hormones. Melting point of $260^{\circ} \mathrm{C}$ for estrone and $178^{\circ} \mathrm{C}$ for $\beta$-estradiol were used.

\subsubsection{SEM images}

The morphology of the synthesized samples: Q-mag-MIP, E1-mag-MIP, E2-mag-MIP and Q-mag-NIPs were characterized using SEM. The examples of SEM images (low - x 500 and high - x 30000 magnification) are shown in Fig 6 . These images reveal that the obtained magnetic products have spherical morphology and a diameter less than $100 \mathrm{~nm}$. Due to their small size, these nanoparticles agglomerate easily.
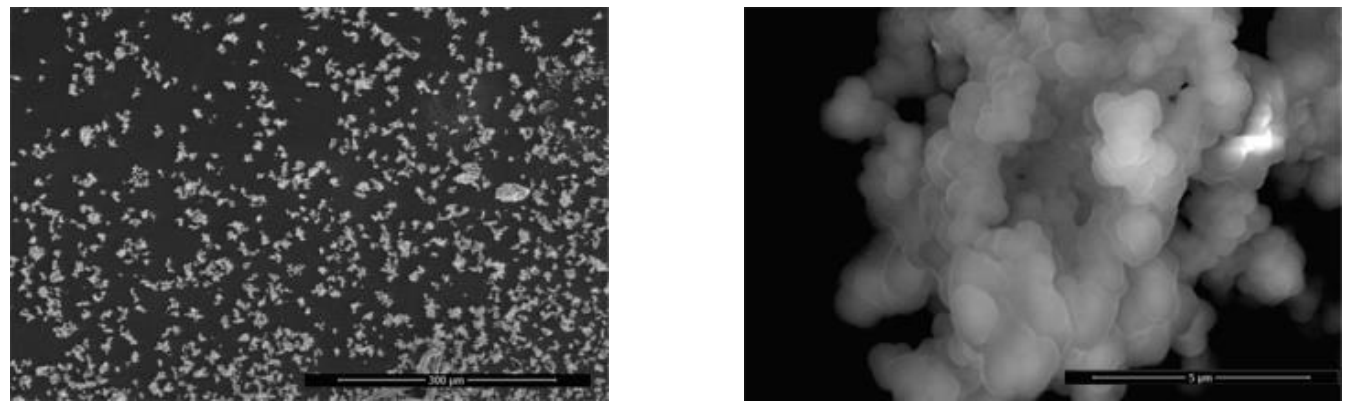

Fig. 6: SEM images of the E2-mag-MIP after template elution with selective cavities to the $\beta$-estradiol.

\subsection{MS analyses}

\subsubsection{ESI-MS}

For quercetin solutions, in the range of positive ions the main signal was found at $\mathrm{m} / \mathrm{z} 303[\mathrm{M}+\mathrm{H}]^{+}$(Fig. 7), while in the range of negative ions - the one at $\mathrm{m} / \mathrm{z} 301[\mathrm{M}-\mathrm{H}]^{-}$. To identify the quercetin presence in the samples, signals related to the molecular peak as well as signals of main fragmentation ions were used. It was found that the intensity of the signal is better for negative ions in concentration of quercetin to $5 \times 10^{-4} \mathrm{M}$, however in low concentrations the signal in the positive ions began to dominate and it is more linear in relation to the concentration. In order to establish the range of applicability of ESI method for determination of quercetin directly from water solutions, a series of measurements were performed. The LOD (MSMS) in the range of positive ions was found at $10^{-8} \mathrm{M}$ and in the range of negative ions at $10^{-7} \mathrm{M}$. In ESI analysis of the lower content of quercetin in water solutions, the probability of making error in measurements or in interpretation of results is lower for the measurements in the range of positive than negative ions. Moreover, on the basis of analysis of the peak at $\mathrm{m} / \mathrm{z} 303$ (range of positive ions), the signal intensity was linearly dependent on the quercetin concentration in the quercetin solution concentration range from $10^{-4}$ to $10^{-7} \mathrm{M}$, which permits quantitative determination of quercetin in this range. Using the ESI-MS method the content of quercetin was determined in water extracts of capers and onion to be 0.00045 $\mathrm{M}$ and $0.000045 \mathrm{M}$, which, calculated on the dry mass of capers and onion gave a quercetin content of $150 \mathrm{mg} / 100 \mathrm{~g}$ and 15 $\mathrm{mg} / 100 \mathrm{~g}$, respectively. For estrone and $\beta$-estradiol solutions in the range of positive ions, the main signal was at $\mathrm{m} / \mathrm{z} 271 \mathrm{and}$ $273[\mathrm{M}+\mathrm{H}]^{+}$. However, the signal intensity was stabled only for a short time (Fig. 8). Despite the use of a mild method of 
ionization electrospray in the MS spectrum, the signals of fragment ions were observed, what make quantitative analysis difficult. The limit of detection ESI-MS in the range of positive ions was $10^{-7} \mathrm{M}$ for estrone and $\beta$-estradiol.

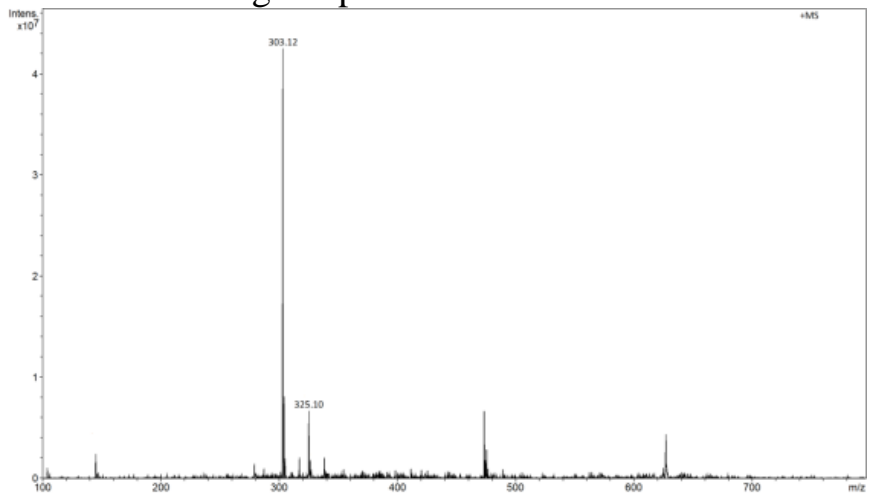

Fig. 7: ESI-MS spectrum of quercetin solution in the ranges of positive ions $\left(\mathrm{m} / \mathrm{z} 303[\mathrm{M}+\mathrm{H}]^{+}\right.$and $\left.325[\mathrm{M}+\mathrm{Na}]^{+}\right)$.

a)

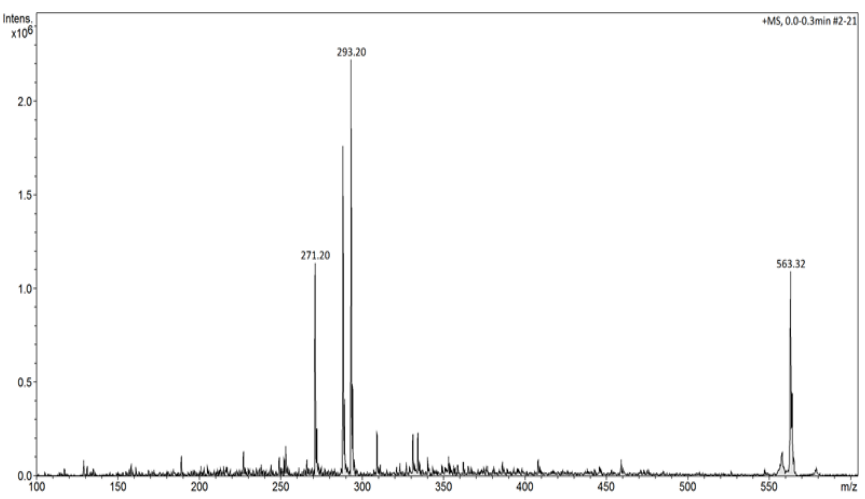

b)

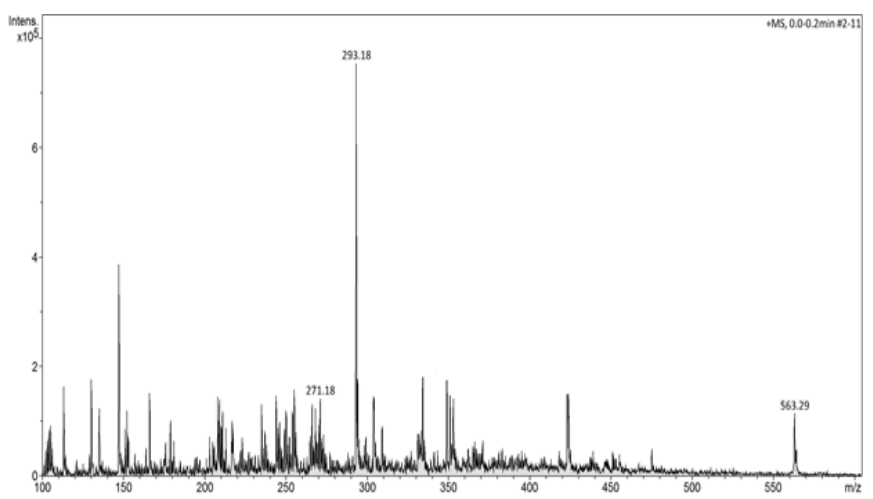

Fig. 8: ESI-MS spectrum of the $10^{-7} \mathrm{M}$ estrone solution in the ranges of positive ions (m/z $271[\mathrm{M}+\mathrm{H}]^{+}$and $\left.293[\mathrm{M}+\mathrm{Na}]^{+}\right)$: a) in $\mathrm{t}=0 \mathrm{~h}$; b) in $\mathrm{t}=24 \mathrm{~h}$.

\subsubsection{FAPA-MS}

In the method of flowing atmospheric pressure afterglow mass spectrometry, the molecules are ionized by the production and flow of thermalized ions in plasma afterglow of helium. When the sample containing the analyte is heated to $350^{\circ} \mathrm{C}$, it liberates the analyte, which is then ionized in a stream of plasma and further gets to the mass spectrometer analyzer. The limit of detection of the analyte in Q-MIPs was $0.005 \mathrm{mg} / \mathrm{g}$ MIP. The procedure for determination of quercetin in water solutions with the use of mag-MIP was as follows: $100 \mathrm{mg}$ of quercetin-selective mag-MIP was added to $10 \mathrm{~mL}$ of the extract containing quercetin. After 30 mins of vigorous stirring, the suspension was centrifuged the obtained Q-mag-MIP system was dried at a temperature below $40^{\circ} \mathrm{C}$. Then, the system was placed on a heating table and analyzed by FAPA-MS. The content of quercetin in solutions was determined up to the concentration of $10^{-9} \mathrm{M}$, following described analytical procedure. The limit of detection FAPA-MS in the range of positive ions was $10^{-9} \mathrm{M}$ and in the range of negative ions is $10^{-8} \mathrm{M}$. Those concentrations of quercetin solutions were assumed as the limits of quantification of the analyte by the Q-mag-MIP-FAPA method (combining of pre-concentration using mag-MIP and FAPA-MS analysis). Described method was applied for determination of quercetin using FAPAMS analysis (Fig. 9), in water solutions of capers and onion. Performed studies indicated on detection limits at 0.00055 and $0.000049 \mathrm{M}$, respectively. Recalculation among the dry mass of capers and onion gave a quercetin content of 158 and $17 \mathrm{mg} / 100 \mathrm{~g}$, respectively. In the case of hormone determination, the same procedure was used. Obtained magnetic polymers sorbed hormones from solutions. Moreover, polymer cavities were selective for particular hormones and made 
them stable within polymeric structures (Fig. 10), which is extremely important for analytical reasons. The combination of analyte pre-concentration technique using molecularly imprinted polymers and FAPA-MS analysis method allowed to reduce the limit of detection for many trace analytes.

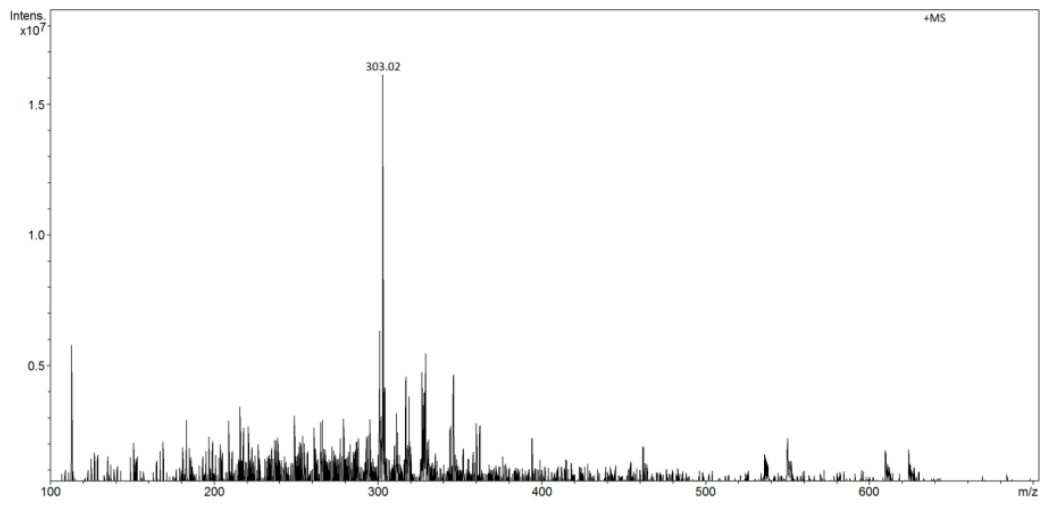

Fig. 9: FAPA-MS spectrum of Q-mag-MIP in the ranges of positive ions $\left(\mathrm{m} / \mathrm{z} 303[\mathrm{M}+\mathrm{H}]^{+}\right)$.

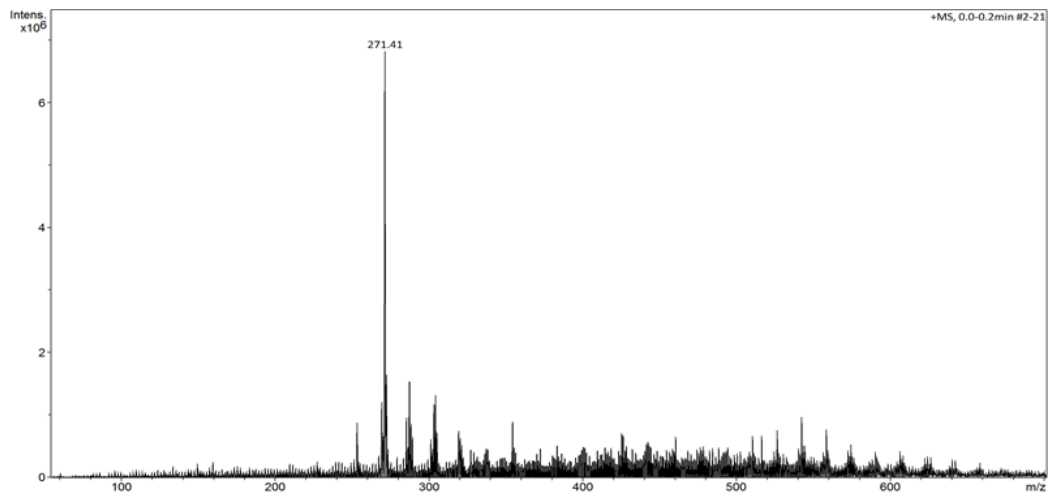

Fig. 10: FAPA-MS spectrum of E1-mag-MIP isolated from $10^{-7} \mathrm{M}$ estrone solution in $\mathrm{t}=24 \mathrm{~h}\left(\mathrm{~m} / \mathrm{z} 271[\mathrm{M}+\mathrm{H}]^{+}\right)$.

\section{Conclusion}

In conclusion, we explored synthesis of different molecularly imprinted polymers coated $\mathrm{Fe}_{3} \mathrm{O}_{4}$ magnetic nanoparticles that exhibit a much higher specific recognition and saturation magnetization. A new method for determination of organic compounds based on the use of mag-MIP dedicated for this analyte and their FAPA-MS analysis was proposed. The range of this method application was established and the results obtained using this method were compared with those achieved for the classical ESI-MS method for determination of estrone, $\beta$-estradiol in water solutions and quercetin of caper juice and onion. Application of the FAPA-MS method, a modern tool enabling one-step, direct testing of organic substances contained in the solid phase, allowed not only for their detection, but also in combination with ESI mass spectrometry techniques for developing of optimal analytical methods for analytes determination in solutions. Comparison of results obtained using both techniques allowed the development of fast analytical methods for the determination of bioactive substances with a molecular weight up to $500 \mathrm{Da}$ in the solid phase.

\section{Acknowledgements}

This work was supported by National Grant no. 2016/21/B/ST4/02082, provided by the National Science Centre. 


\section{References}

[1] R. J. Uzuriaga-Sanchez, S. Khan, A. Wong, G. Picasso, M. I. Pividori and M. del Pilar Taboada Sotomayor, "Magnetically separable polymer (Mag-MIP) for selective analysis of biotin in food samples," Food Chemistry, no. 190, pp. 460-467, 2016.

[2] J. Wackerlig and R. Schirhagl, "Applications of Molecularly Imprinted Polymer Nanoparticles and Their Advances toward Industrial Use: A Review," Analytical Chemistry, vol. 88, no. 1, pp. 250-261, 2015.

[3] P. A. G. Cormack and A. Zurutuza Elorza, "Molecularly imprinted polymers: synthesis and characterisation," Journal of Chromatography B, vol. 804, no. 1, pp. 173-182, 2004.

[4] X. Wang, L. Wang, X. He, Y. Zhang and L. Chen, "A molecularly imprinted polymer-coated nanocomposite of magnetic nanoparticles for estrone recognition," Talanta, vol. 78, no. 2, pp. 327-332, 2009.

[5] H. Lu and S. Xu, "Mesoporous structured estrone imprinted Fe3O4@SiO2@mSiO2 for highly sensitive and selective detection of estrogens from water samples by HPLC," Talanta, vol. 144, no. 1, pp. 303-311, 2015.

[6] Q. Han, X. Shen, W. Zhu, C. Zhu, X. Zhou and H. Jiang, "Magnetic sensing film based on Fe3O4@ Au-GSH molecularly imprinted polymers for the electrochemical detection of estradiol," Biosensors and Bioelectronics, vol. 79, no. 1, pp. 180-186, 2016.

[7] M. Guć and G. Schroeder, "The Molecularly Imprinted Polymers. Influence of Monomers on The Properties of Polymers -A Review," World Journal of Research and Review, vol. 5, no. 6, pp. 36-47, 2017.

[8] S. Ghafoor and S. Ata, "Synthesis of carboxyl-modified $\mathrm{Fe}_{3} \mathrm{O}_{4} @ \mathrm{SiO}_{2}$ nanoparticles and their utilization for the remediation of cadmium and nickel from aqueous solution," Journal of the Chilean Chemical Society, vol. 62, no. 3, pp. 3588-3592, 2017.

[9] M. Kopperi and M. L. Riekkola, "Non-Targeted Evaluation of Selectivity of Water-Compatible Class Selective Adsorbents for the Analysis of Steroids in Wastewater," Analytica Chimica Acta, vol. 920, pp. 47-53, 2016.

[10] M. J. Alda and D. Barceló, "Review of Analytical Methods for the Determination of Estrogens and Progestogens in Waste Waters," Analytical and Bioanalytical Chemistry, vol. 371, no.4, pp. 437-47, 2019.

[11] V. Pichon and F. Chapuis-Hugon, "Role of Molecularly Imprinted Polymers for Selective Determination of Environmental Pollutants-A Review," Analytica Chimica Acta, vol. 622, no. 1-2, pp. 48-61, 2008.

[12] M. Cegłowski, M. Smoluch, E. Reszke, J. Silberring and G. Schroeder, "Molecularly imprinted polymers as selective adsorbents for ambient plasma mass spectrometry," Analytical and Bioanalytical Chemistry, vol. 409, no. 13, pp. 339333405, 2017. 